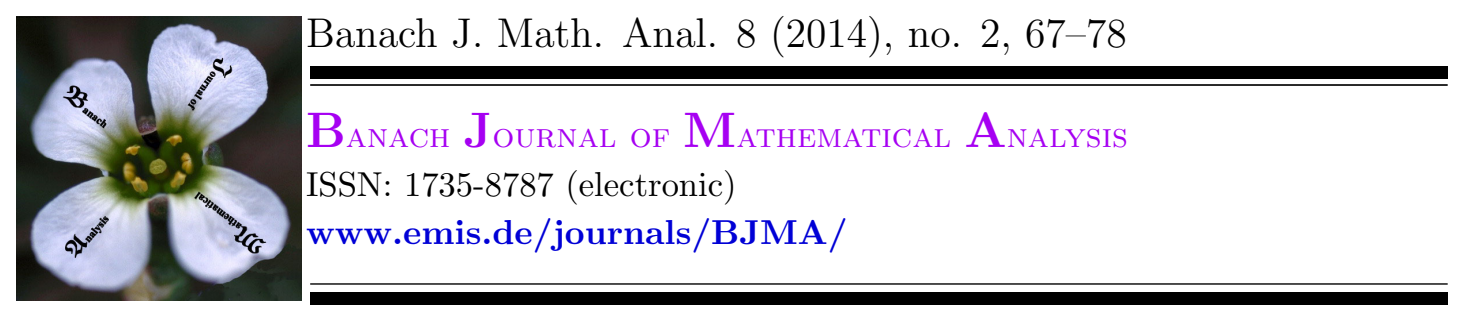

\title{
THE CONTROLLED SEPARABLE COMPLEMENTATION PROPERTY AND MONOLITHIC COMPACTA
}

\author{
JESÚS FERRER
}

Communicated by J. E. Ball

\begin{abstract}
For a compact $K$, a necessary condition for $C(K)$ to have the Controlled Separable Complementation Property is that $K$ be monolithic. In this paper, we prove that when $K$ contains no copy of $\left[0, \omega^{\omega}\right]$ and the set of points which admit a countable neighborhood base is a cofinite subset of $K$, then monolithicity of $K$ is sufficient for $C(K)$ to enjoy the Controlled Separable Complementation Property. We also show that, for this type of compacta $K$, the space $C(K)$ is separably extensible.
\end{abstract}

\section{INTRODUCTION AND PRELIMINARIES}

We began to study the Controlled Separable Complementation Property (which in what follows we shall refer to as $C S C P$, to shorten up) when, back in 2002, R. M. Aron posed us the problem of studying conditions on a real Banach space under which one could have that every continuous homogeneous polynomial would have a zero-set containing an infinite-dimensional linear subspace. In particular, it was conjectured in [2] that if a real Banach space $X$ is not injected into a Hilbert space, i.e., no one-to-one bounded linear map can be defined from $X$ into a Hilbert space, then the zero-set of each continuous quadratic polynomial must contain a non-separable linear subspace. In [4], this conjecture was shown to be true for spaces having the $C S C P$, and this is how we started getting involved with this property.

Date: Received: May 10, 2013; Revised: Sep. 5, 2013; Accepted: Sep. 9, 2013.

2010 Mathematics Subject Classification. Primary 46B10; Secondary 46B26.

Key words and phrases. Controlled separable complementation property, monolithic compacta, extensible space. 
A Banach space $X$ (always real) is said to possess the $C S C P$ if, for every two separable subspaces $E$ and $F$ of $X$ and $X^{*}$, respectively, there is a bounded projection $P$ on $X$ such that

(i) $P(X)$ is separable,

(ii) $E \subseteq P(X)$,

(iii) $F \subseteq P^{*}\left(X^{*}\right)$.

The notion of CSCP was introduced in [14] to study the structure of certain quotients of Banach spaces, although it was refered to as the Controlled Separable Projection Property. There is a wide class of Banach spaces which have this property, in fact, after the characterization of the Weakly Lindelöf Determined spaces $(W L D)$ given in [9], it can be deduced that the class of spaces having the $C S C P$ contains the $W L D$. Since we showed in [6] that the space $C\left[0, \omega_{1}\right]$ has the $C S C P$, it follows that the inclusion between those two classes is strict. Although we still do not know whether the $C S C P$ is inherited by closed linear subspaces in general, it was also proved in the above reference that countable intersections of closed hyperplanes do inherit the property. Thus, the closed hyperplane of $C\left[0, \omega_{1}\right]$ formed by the elements which vanish in $\omega_{1}$ has the $C S C P$. It may be interesting to notice that this hyperplane does not admit a Projectional Resolution of the Identity $(P R I)$, [5]. Let us also mention that there are other examples of $C(K)$ spaces (with $K$ linearly ordered) having the $C S C P$ and being non-isomorphic to any space with a $P R I,[11$, Th. 18.4].

If $X$ has the $C S C P$, it is not hard to see, [7], that the dual unit ball $B_{X^{*}}$ is monolithic respect to the weak-star topology. Let us just say that the notion of a monolithic space is due to Arkhangel'skii, [1]. A compact $K$ is said to be monolithic whenever each separable subset is second countable, by Uryshon's metrization theorem, this equals to say that each separable subset must be metrizable. Probably, the best known class of this type of spaces is the one formed by Corson compacta. As a consequence of what we stated before, since monolithicity is hereditary, if $C(K)$ has the $C S C P$ then $K$ has to be monolithic.

Let us first admit our incapability in finding a monolithic compact $K$ whose $C(K)$ space does not have the $C S C P$, i.e., we do not know wether monolithicity of $K$ alone implies the $C S C P$ for $C(K)$. Therefore, the main purpose of this paper is to study under what extra conditions monolithicity of $K$ will be sufficient to guarantee that $C(K)$ has the $C S C P$. It may be interesting to recall that in [12] it has been shown that for $K$ a compact line the above stated problem has a positive answer, i.e., $C(K)$ has the $C S C P$ if and only if $K$ is monolithic. Also, in [7] and [8], it is proved that the same can be said when $K$ is a Mrówka compact. As a matter of fact, what we obtain here is an extension of this last result.

Given an infinite set $S$, two subsets $A, B$ of $S$ are said to be almost disjoint whenever $A \cap B$ is a finite set. Let $\mathbf{A}$ be a family formed by almost disjoint countably infinite subsets of $S$. In $S \cup \mathrm{A}$ we define the following topology: The points of $S$ are isolated and, for each $A \in \mathbf{A}$, a basic neighborhood of $A$ has the form $\{A\} \cup B$, where $B$ is a cofinite subset of $A$. It is plain that, with this topology, $S \cup \mathbf{A}$ is a scattered locally compact space. Its one-point compactification $K_{\mathbf{A}}:=$ $S \cup \mathbf{A} \cup\{\infty\}$ is known as a Mrówka compact. 
For a compact $K$, we say that a point $s \in K$ is a locally first countable point provided there is an open neighborhood $V$ of $s$ such that $V$ is first countable. Denoting by $F_{K}$ the set of points of $K$ which are not locally first countable, it is clear that $F_{K}$ is a closed subset of $K$. We say that a compact $K$ is locally almost first countable whenever the set $F_{K}$ is finite. Notice that all Mrówka compacta are locally almost first countable.

A Banach space $X$ is said to be separably extensible whenever, for each separable closed subspace $Y$ of $X$ and each bounded linear map $S: Y \rightarrow X$, there is a bounded linear map $T: X \rightarrow X$ such that $T_{\mid Y}=S$. Whenever bounded linear maps on arbitrary closed subspaces of a Banach space admit a bounded extension to the whole space, then the space is said to be extensible, see [13]. In [3], an implicit demand for scattered compacta $K$ whose $C(K)$ space may be extensible but not isomorphic to any $c_{0}(\Gamma)$ is made. Although we have not been able to give a definite answer to this demand, we shall show that there is a whole class of such compacta whose space of continuous functions is separably extensible. This class contains all monolithic non-Eberlein Mrówka compacta, in particular the Mrówka compacta induced by the so called non-trivial ladder systems, see [7]. In the last section, we give an example of one of such Mrówka compacta $K_{\mathbf{L}}$ such that $C\left(K_{\mathbf{L}}\right)$ is not isomorphic to any $c_{0}(\Gamma), C\left(K_{\mathbf{L}}\right)$ is separably extensible, but it is not extensible since it contains complemented and uncomplemented copies of $c_{0}\left(\omega_{1}\right)$.

\section{Monolithicity versus the $C S C P$}

We begin this section by stating its main result

Theorem 2.1. Let $K$ be a locally almost first countable compact such that it contains no copy of $\left[0, \omega^{\omega}\right]$. Then, $C(K)$ has the Controlled Separable Complementation Property if and only if $K$ is monolithic.

In order to give the proof of the above theorem, since necessity is always true, we only need showing its sufficiency. Probably, the most determining fact which led us to try to achieve the former theorem was learning that a monolithic compact with no copies of $\left[0, \omega^{\omega}\right]$ has to be scattered and of finite height. This result, with a quite clear and elegant proof, can be found in [3]. Thus, we just have to show that, for $K$ a locally almost first countable monolithic scattered compact of finite height, $C(K)$ has the $C S C P$.

If $S$ is a topological space and $\alpha$ is an ordinal number, the $\alpha$-th derivative of $S$ is defined inductively as follows: $S^{(0)}=S, S^{(\alpha+1)}=S^{(\alpha)}$ ' , i.e. the set of accumulation points of $S^{(\alpha)}$, finally $S^{(\alpha)}=\cap_{\beta<\alpha} S^{(\beta)}$, when $\alpha$ is a limit ordinal. The smallest ordinal $\alpha$ for which $S^{(\alpha+1)}=S^{(\alpha)}$ is called the Cantor-Bendixson height of $S$. For a scattered compact $K$, we know that there is a certain ordinal $\alpha$ such that $K^{(\alpha)}=\emptyset$, thus the Cantor-Bendixson height of $K$ coincides with the smallest ordinal $\alpha$ for which $K^{(\alpha)}=\emptyset$; consequently, if $K$ has a Cantor-Bendixson height, which is non-limit, say $\alpha+1$, then the set $K^{(\alpha)}$ is a non-empty finite set. 
In what follows, when $K$ is a scattered compact of height $n+1$, we use the following notation. By defining

$$
M_{1}:=K \backslash K^{(1)}, \quad M_{2}:=K^{(1)} \backslash K^{(2)}, \cdots, M_{n}:=K^{(n-1)} \backslash K^{(n)},
$$

we have that

$$
K=M_{1} \cup M_{2} \cup \cdots \cup M_{n} \cup K^{(n)},
$$

where all these sets are pairwise disjoint. Clearly, it is important to recall that for a scattered compact being monolithic equals saying that the closure of each countable subset is also countable. If $A$ is a subset of $K$, we write $C_{A}(K)$ to represent the closed subspace of $C(K)$ formed by all elements which vanish in $A$. Although the following auxiliary lemma may be well-known, we prove it here for the sake of completeness.

Lemma 2.2. Let $K$ be a locally almost first countable scattered compact of height $n+1$. Then each point $s \in K \backslash F_{K} \backslash K^{(n)}$ admits a countable clopen neighborhood $V(s)$, contained in $K \backslash F_{K} \backslash K^{(n)}$, such that, if $s \in M_{i}$ for some $i \in\{1,2, \cdots, n\}$, then

$$
V(s) \cap\left(M_{i} \cup M_{i+1} \cup \cdots \cup M_{n} \cup K^{(n)}\right)=\{s\} .
$$

Proof. We proceed inductively over the height of $K$. For height of $K$ being 2, since $K=M_{1} \cup K^{(1)}$ and $M_{1}$ consists of isolated points, for each $s \in M_{1},\{s\}$ is a clopen neighborhood of $s$ satisfying all our requirements. Assuming the statement is true for any scattered compact of height less or equal to $n$ satisfying all our conditions, we show that it also holds for this type of compact $K$ when its height is $n+1$.

Let $s \in K \backslash F_{K} \backslash K^{(n)}$. Since, for $s \in M_{1}$, again the clopen set $\{s\}$ fulfills all our conditions, we assume that $s \in M_{i}$, for some $i \in\{2,3, \cdots, n\}$. Since $K \backslash F_{K} \backslash K^{(n)}$ is open and first countable, there is a decreasing sequence $\left\{V_{j}: j \geq 1\right\}$ of clopen neighborhoods of $s$ (notice that a scattered compact is always zero-dimensional) such that it is a neighborhood base for $s, V_{j} \subseteq K \backslash F_{K} \backslash K^{(n)}, j \geq 1$, and also, from the definition of the sets $M_{1}, M_{2}, \cdots, M_{n}$,

$$
V_{j} \cap\left(M_{i} \cup M_{i+1} \cup \cdots \cup M_{n}\right)=\{s\}, \quad j \geq 1 .
$$

Noticing that

$$
V_{1}=\left(V_{1} \cap M_{1}\right) \cup\left(V_{1} \cap M_{2}\right) \cup \cdots \cup\left(V_{1} \cap M_{i-1}\right) \cup\{s\}
$$

is a scattered compact of height $i \leq n$ such that $F_{V_{1}}=\emptyset$, we have that $V_{1}$ is a first countable scattered compact of finite height. It is well-known that this type of compacta are countable. Taking $V(s):=V_{1}$, we are done.

Lemma 2.3. For $K$ as before, each closed set of $K$ contained in $K \backslash F_{K}$ is countable.

Proof. Let $S$ be a closed set of $K$ which is is contained in $K \backslash F_{K}$. Since $S=\left(S \backslash K^{(n)}\right) \cup\left(S \cap K^{(n)}\right)$, making use of Lemma 2.2 and its notation plus the fact that, for each point $s \in S \cap K^{(n)}$, there is a clopen neighborhood $W(s)$ of $s$ 
which is first countable, hence countable after being scattered with finite height, we have that the collection

$$
\{K \backslash S\} \cup\left\{V(s): s \in S \backslash K^{(n)}\right\} \cup\left\{W(s): s \in S \cap K^{(n)}\right\}
$$

is an open cover of $K$ and so there are $s_{1}, s_{2}, \cdots, s_{p}$ in $S \backslash K^{(n)}$ and $s_{p+1}, \cdots, s_{q}$ in $S \cap K^{(n)}$ such that $S \subseteq V\left(s_{1}\right) \cup V\left(s_{2}\right) \cup \cdots \cup V\left(s_{p}\right) \cup W\left(s_{p+1}\right) \cup \cdots \cup W\left(s_{q}\right)$. This shows that $S$ must be countable.

Lemma 2.4. Let $K$ be as before. If $K$ is monolithic, then, given a countable subset $N$ of $K \backslash F_{K} \backslash K^{(n)}$, there is a countable open set $G \subseteq K \backslash F_{K} \backslash K^{(n)}$ such that the closure $\bar{G} \subseteq G \cup F_{K} \cup K^{(n)}$ and $G$ contains $N$.

Proof. Again we proceed by induction over the height $n+1$. Since for height 2 the statement holds trivially, we assume that it is true for scattered compacta of height $n$ satisfying our conditions and we prove that it is also true for a scattered compact $K$ of height $n+1$ with the above properties.

Let $N$ be a countable subset of $\left(M_{1} \cup M_{2} \cup \cdots \cup M_{n}\right) \backslash F_{K}$. Since $K$ is monolithic, the closure $\bar{N}$ is countable and so is $\bar{N} \cap\left(M_{2} \cup M_{3} \cup \cdots \cup M_{n}\right) \backslash F_{K}$. Now, this is a countable subset of the scattered compact of height $n \quad K_{0}:=\left(M_{2} \cup M_{3} \cup\right.$ $\left.\cdots \cup M_{n}\right) \cup K^{(n)}$, which satisfies all our requirements. Hence, since $F_{K_{0}} \subseteq F_{K}$ and $K_{0}^{(n)}=K^{(n)}$, there is a countable subset $H$ of $\left(M_{2} \cup M_{3} \cup \cdots \cup M_{n}\right) \backslash F_{K_{0}}$, which is open in $K_{0}$, such that

$$
\begin{gathered}
\bar{N} \cap\left(M_{2} \cup M_{3} \cup \cdots\right. \\
\left.\cup M_{n}\right) \backslash F_{K} \subseteq \bar{N} \cap\left(M_{2} \cup M_{3} \cup \cdots \cup M_{n}\right) \backslash F_{K_{0}} \subseteq H \subseteq \bar{H}=\bar{H}^{K_{0}} \\
\subseteq H \cup F_{K_{0}} \cup K^{(n)} \subseteq H \cup F_{K} \cup K^{(n)} .
\end{gathered}
$$

For $i=2,3, \cdots, n$, we write $H \cap M_{i}$ as the sequence (possibly finite)

$$
H \cap M_{i}=\left\{s_{i j}: j \geq 1\right\},
$$

and so

$$
H=\bigcup_{i=2}^{n}\left\{s_{i j}: j \geq 1\right\}
$$

Since $K \backslash F_{K} \backslash K^{(n)}$ is first countable, we know from Lemma 2.2 that each element $s \in K \backslash F_{K} \backslash K^{(n)}$ admits a clopen neighborhood $V(s)$, contained in $K \backslash F_{K} \backslash K^{(n)}$, which is countable and such that, if $s \in M_{i}$ for some $i \in\{1,2, \cdots, n\}$, then

$$
V(s) \cap\left(M_{i} \cup M_{i+1} \cup \cdots \cup M_{n} \cup K^{(n)}\right)=\{s\} .
$$

Also, since $H$ is open in $K_{0}$, there is an open set $W$ in $K$ such that $W \cap$ $K_{0}=H$. Thus, for each $j \geq 1$, there are countable clopen neighborhoods $W\left(s_{2 j}\right), W\left(s_{3 j}\right), \cdots, W\left(s_{n j}\right)$ of $s_{2 j}, s_{3 j}, \cdots, s_{n j}$, respectively, each one of them contained in $W \cap V\left(s_{2 j}\right), W \cap V\left(s_{3 j}\right), \cdots, W \cap V\left(s_{n j}\right)$, respectively. Let

$$
V:=\bigcup_{j \geq 1}\left(W\left(s_{2 j}\right) \cup W\left(s_{3 j}\right) \cup \cdots \cup W\left(s_{n j}\right)\right) .
$$

Then, $V$ is a countable set and we may write

$$
\bar{V} \backslash F_{K} \backslash K^{(n)}=\left(V \cap M_{1}\right) \cup\left(\bar{V} \cap M_{2} \backslash F_{K}\right) \cup \cdots \cup\left(\bar{V} \cap M_{n} \backslash F_{K}\right),
$$


with, for $i=2,3, \cdots, n$,

$$
\bar{V} \cap M_{i} \backslash F_{K}=\left\{s_{i j}: j \geq 1\right\} \cup\left\{t_{i j}: j \geq 1\right\},
$$

where, for $i=2,3, \cdots, n$, the set $\left\{t_{i j}: j \geq 1\right\}$ is a (possibly finite) subset of $M_{i} \backslash F_{K}$ which is disjoint with $\left\{s_{i j}: j \geq 1\right\}$. Notice that, since $\bar{H} \backslash F_{K} \backslash K^{(n)} \subseteq H$, for each $i=2,3, \cdots, n$ and each $j \geq 1, t_{i j} \notin \bar{H}$. Consequently, there is a clopen neighborhood $U\left(t_{i j}\right)$ of $t_{i j}$ such that

$$
U\left(t_{i j}\right) \cap H=\emptyset \text {. }
$$

For $i=2,3, \cdots, n$, we define inductively the following sets

$$
\left\{\begin{array}{l}
C_{i 1}:=W\left(s_{i 1}\right) \backslash F_{K}, \\
C_{i j}:=W\left(s_{i j}\right) \backslash\left(\bigcup_{h=2}^{n}\left(U\left(t_{h 1}\right) \cup U\left(t_{h 2}\right) \cup \cdots \cup U\left(t_{h j-1}\right)\right)\right) \backslash F_{K}, \quad j \geq 2 .
\end{array}\right.
$$

For $i=2,3, \cdots, n$, setting $C_{i}:=\cup_{j \geq 1} C_{i j}$, we have that each $C_{i}$ is a subset of $V$ and so it is countable. Let

$$
G:=\overline{N \cup C_{2} \cup C_{3} \cup \cdots \cup C_{n}} \backslash F_{K} \backslash K^{(n)} .
$$

Then, after the monolithicity, $G$ is countable, $N \subseteq G, \bar{G} \subseteq G \cup F_{K} \cup K^{(n)}$. To finish the job, we must show that $G$ is open in $K$ :

Observing that $G=\left(G \cap M_{1}\right) \cup\left(G \cap M_{2}\right) \cup \cdots \cup\left(G \cap M_{n}\right)$ and that $G \cap M_{1}$ is clearly open, it all reduces to see that $G$ is a neighborhood of each point in $G \cap M_{i}, 2 \leq i \leq n$. But, for $i=2,3, \cdots, n$,

$$
\begin{aligned}
G \cap M_{i} & =\left(\bar{N} \cup \overline{C_{2}} \cup \overline{C_{3}} \cup \cdots \cup \overline{C_{n}}\right) \cap M_{i} \backslash F_{K} \subseteq\left(H \cap M_{i}\right) \cup\left(\bar{V} \cap M_{i} \backslash F_{K}\right) \\
& =\left\{s_{i j}: j \geq 1\right\} \cup\left\{t_{i j}: j \geq 1\right\},
\end{aligned}
$$

and, for each $j \geq 1$, the set

$$
U:=U\left(t_{i j}\right) \backslash\left(\bigcup_{h=2}^{n}\left(W\left(s_{h 1}\right) \cup W\left(s_{h 2}\right) \cup \cdots \cup W\left(s_{h j}\right)\right) \backslash \bar{N}\right.
$$

is a neighborhood of $t_{i j}$ (notice that $t_{i j} \notin \bar{N}$ and $t_{i j} \notin W\left(s_{h k}\right), 2 \leq h \leq n$, $1 \leq k \leq j$, otherwise $\left.t_{i j} \in W \cap M_{i} \subseteq H\right)$. Consequently, since for $2 \leq h \leq n$, $k>j, \bar{U} \cap C_{h k}=\emptyset$,

$$
\begin{gathered}
U \cap\left(N \cup C_{2} \cup C_{3} \cup \cdots \cup C_{n}\right)=U \cap\left(\bigcup_{h=2}^{n}\left(C_{h 1} \cup C_{h 2} \cup \cdots \cup C_{h j}\right)\right. \\
\subseteq U \cap\left(\bigcup_{h=2}^{n} W\left(s_{h 1}\right) \cup W\left(s_{h 2}\right) \cup \cdots \cup W\left(s_{h j}\right)\right)=\emptyset
\end{gathered}
$$

and this shows that $t_{s j} \notin G$, i.e.,

$$
G \cap M_{i}=\left\{s_{i j}: j \geq 1\right\} .
$$

Now, for each $j \geq 1, C_{i j}$ is a neighborhood of $s_{i j}$ contained in $G$, therefore $G$ is a neighborhood of each point in $G \cap M_{i}$ as we wanted.

Proposition 2.5. If $K$ is a monolithic scattered compact of finite height, then $C_{F_{K}}(K)$ has the $C S C P$. 
Proof. Assuming the height of $K$ is $n+1$, using the terminology previously introduced, consider the finite-codimensional closed subspace of $C_{F_{K}}(K)$ given by

$$
C_{F_{K} \cup K^{(n)}}(K):=\left\{x \in C(K): x_{F_{F_{K} \cup K^{(n)}}}=0\right\} .
$$

It all reduces to show that $C_{F_{K} \cup K^{(n)}}(K)$ has the $C S C P$. Thus, let $\left\{x_{j}: j \geq\right.$ $1\}$ and $\left\{\mu_{j}: j \geq 1\right\}$ be countable subsets of $C_{F_{K} \cup K^{(n)}}(K)$ and $C_{F_{K} \cup K^{(n)}}(K)^{*}$, respectively. For each $j, h \geq 1$, the set $A_{j h}:=\left\{s \in K:\left|x_{j}(s)\right| \geq \frac{1}{h}\right\}$ is a closed subset of $K$ contained in $K \backslash F_{K} \backslash K^{(n)}$, which after Lemma 2.3 is countable. Also, after the monolithicity, since, for each $j \geq 1, \mu_{j}$ is a purely atomic Radon measure in $K$, its support supp $\mu_{j}$ is a countable subset of $K$, let $B_{j}:=\operatorname{supp} \mu_{j} \backslash F_{K} \backslash K^{(n)}$. Therefore, setting $N:=\left(\cup_{j, h \geq 1} A_{j h}\right) \cup\left(\cup_{j \geq 1} B_{j}\right)$, we obtain a countable subset $N \subseteq K \backslash F_{K} \backslash K^{(n)}$ such that, for each $j \geq 1$,

$$
x_{j_{\mid K \backslash N}}=0, \quad \operatorname{supp} \mu_{j} \subseteq N \cup F_{K} \cup K^{(n)} .
$$

From Lemma 2.4, there is a countable open subset $G$ of $K \backslash F_{K} \backslash K^{(n)}$ such that

$$
N \subseteq G \subseteq \bar{G} \subseteq G \cup F_{K} \cup K^{(n)}
$$

Defining

$$
P x:=x \cdot 1_{G}, \quad x \in C_{F_{K} \cup K^{(n)}}(K),
$$

we obtain a well-defined bounded projection $P$ on $C_{K \cup K^{(n)}}(K)$ such that $G \subseteq$ (Ker $P)^{\perp}$. Since $\bar{G}$ is metrizable we have that $P\left(C_{K \cup K^{(n)}}(K)\right)$ is separable. It is straightforward to check that $\left\{x_{j}: j \geq 1\right\}$ is contained in $P\left(C_{K \cup K^{(n)}}(K)\right)$ and also that $\left\{\mu_{j}: j \geq 1\right\}$ is contained in $(\operatorname{Ker} P)^{\perp}=P^{*}\left(C_{K \cup K^{(n)}}(K)^{*}\right)$.

Corollary 2.6. If $K$ is a monolithic scattered compact of finite height such that it is locally almost first countable, then $C(K)$ has the $C S C P$.

Proof of Theorem 2.1. As we said before, sufficiency is the only part needing be proved. If $K$ is a monolithic compact with no copies of $\left[0, \omega^{\omega}\right]$, then, after [3, Proposition 3], we have that $K$ is scattered with finite height. If $K$ is also locally almost first countable, the former corollary now applies.

As mentioned in the introduction, we do not know if in general monolithicity of a compact $K$ alone is enough to guarantee that $C(K)$ has the $C S C P$, even the same problem for scattered compacta is still open. Therefore, after our former result, it may be interesting to notice that there are monolithic scattered compacta which are not locally almost first countable. For this, just consider $K$ as the set of all characteristic functions of at most 2-element subsets of an uncountable set, endowed with the product topology. Obviously, $K$ is Eberlein and therefore monolithic; on the other hand, it has uncountably many points of uncountable character. 


\section{A Class of SEPARABly EXTENSIBle SPACES}

This last section is devoted to show that the $C(K)$ spaces corresponding to the class of compacta $K$ appearing in the previous section have the property of being separably extensible. Hence, the main result of this part reads as follows

Theorem 3.1. Let $K$ be a locally almost first countable compact such that it contains no copy of $\left[0, \omega^{\omega}\right]$. If $K$ is monolithic, then $C(K)$ is separably extensible.

In order to prove the above stated theorem, we start by showing a general result.

Proposition 3.2. Let $X$ be a Banach space such that every separable subspace is contained in a complemented copy of $c_{0}$. Then $X$ is separably extensible.

Proof. Let $S: Y \rightarrow X$ be a bounded linear map defined in the separable closed subspace $Y$ of $X$. Since $Y \cup S(Y)$ is separable, by hypothesis, there is a closed subspace $Z$ of $X$, which is complemented in $X$ and isomorphic to $c_{0}$, such that $Y \cup S(Y) \subseteq Z$. Let $P$ be the bounded projection on $X$ such that $P(X)=Z$ and let $T_{1}: Z \rightarrow c_{0}$ be a topological isomorphism. The map $S_{0}: T_{1}(Y) \rightarrow c_{0}$ such that, for each $y \in Y, S_{0}\left(T_{1} y\right):=T_{1}(S y)$, is well-defined linear and bounded. Since it is well known that $c_{0}$ is an extensible space, there is a bounded linear map $S_{1}: c_{0} \rightarrow c_{0}$ such that $S_{1_{\mid T_{1}(Y)}}=S_{0}$. Finally, defining $T:=T_{1}^{-1} S_{1} T_{1} P$, we obtain a well-defined map which is linear bounded and we show that it extends $S$. For each $y \in Y$,

$$
T y=\left(T_{1}^{-1} S_{1} T_{1} P\right) y=T_{1}^{-1}\left(S_{0} T_{1} y\right)=T_{1}^{-1}\left(T_{1} S y\right)=S y .
$$

Proposition 3.3. Let $K$ be a monolithic locally almost first countable compact space with no copies of $\left[0, \omega^{\omega}\right]$. Then every separable subspace of $C(K)$ is contained in a complemented isomorphic copy of $c_{0}$.

Proof. The compact $K$ is a scattered one with finite height, say of height $n+1$. Hence, using the terminology settled formerly, since $F_{K} \cup K^{(n)}$ is a finite set, it will be sufficient to see that each separable subspace $Y$, which we already assume has infinite dimension, of the finite-codimensional space $X:=C_{F_{K} \cup K^{(n)}}(K)$ is contained in a complemented copy of $c_{0}$. After the proof of Proposition 2.5, we know that there is a countably infinite open set $G$, contained in $K \backslash F_{K} \backslash K^{(n)}$, whose closure in $K$ is contained in $G \cup F_{K} \cup K^{(n)}$, which provides with a bounded projection

$$
\begin{aligned}
P: & X \longrightarrow X \\
x & \longrightarrow x \cdot 1_{G},
\end{aligned}
$$

such that $Y \subseteq P(X)$. But $P(X)$ is isomorphic to the space $C_{F_{K} \cup K^{(n)}}(\bar{G})$, which is a finite-codimensional subspace of $C(\bar{G})$. Now, since $\bar{G}$ is countable and of finite height, it is clear that $C(\bar{G})$ is isomorphic to $c_{0}$, and so is $P(X)$.

Proof of Theorem 3.1. If $K$ is a monolithic locally almost first countable compact with no copies of $\left[0, \omega^{\omega}\right]$, then, from Proposition 3.3 , we have that every 
separable subspace of $C(K)$ is contained in a complemented isomorphic copy of $c_{0}$. Proposition 3.2 implies that $C(K)$ is separably extensible.

In the following we give an example of a non-trivial $\omega_{1}$-ladder system compact $K_{\mathbf{L}}$, which is therefore monolithic non-Eberlein, whose space $C\left(K_{\mathbf{L}}\right)$ is separably extensible although it is not extensible. Let us recall that an $\omega_{1}$-ladder system is an almost disjoint family of countable subsets of $\omega_{1}$ of the form

$$
\mathbf{L}=\left\{L_{\delta}: \delta \in S\right\},
$$

where $S$ is a subset of $\omega_{1}^{(1)}$, the set of order-accumulation points of $\omega_{1}$, and, for each $\delta \in S$,

$$
L_{\delta}=\left\{\alpha_{\delta, j}: j \in \omega\right\}
$$

is a strictly increasing sequence in $\omega_{1}$ such that $\sup _{j} \alpha_{\delta, j}=\delta$. When the set $S$ is stationary, i.e., it meets every closed unbounded (club) subset of $\omega_{1}$, see [10], then its associated ladder system $\mathbf{L}$ is said to be non-trivial. In [7], it is shown that the Mrówka compact $K_{\mathbf{L}}$ is always monolithic, while it is Eberlein if and only if $\mathbf{L}$ is trivial.

Example 3.4. By $\omega_{1}^{(2)}$ we represent the set of order-accumulation points of $\omega_{1}^{(1)}$. For each ordinal $\delta \in \omega_{1}^{(1)} \backslash \omega^{(2)}$, let $L_{\delta}$ be a strictly increasing sequence of elements of $\omega_{1} \backslash \omega_{1}^{(1)}$ whose supremum is $\delta$. If $\delta \in \omega^{(2)}$, let $L_{\delta}$ be a strictly increasing sequence of elements of $\omega_{1}^{(1)}$ whose supremum is $\delta$. The collection $\mathbf{L}:=\left\{L_{\delta}\right.$ : $\left.\delta \in \omega_{1}^{(1)}\right\}$ is an $\omega_{1}$-ladder system, hence its associated Mrówka compact $K_{\mathbf{L}}$ is monolithic and non-Eberlein, since $\mathbf{L}$ is non-trivial.

Consider now the Mrówka compact given by, if $\mathbf{L}_{0}:=\left\{L_{\delta}: \delta \in \omega_{1}^{(1)} \backslash \omega_{1}^{(2)}\right\}$,

$$
K_{0}:=\left(\cup \mathbf{L}_{0}\right) \cup\left\{l_{L}: L \in \mathbf{L}_{0}\right\} \cup\{\infty\} .
$$

Then, since the set $\omega_{1}^{(1)} \backslash \omega_{1}^{(2)}$ is non-stationary, we have that $K_{0}$ is Eberlein and so $C\left(K_{0}\right)$, as shown in [7], is isomorphic to $c_{0}\left(\omega_{1}\right)$. We show that $K_{0}$ is a retract of $K_{\mathbf{L}}$, hence $C\left(K_{\mathbf{L}}\right)$ will contain a complemented isomorphic copy of $c_{0}\left(\omega_{1}\right)$. To do this, define the map $\varphi: K_{\mathbf{L}} \rightarrow K_{\mathbf{L}}$ as, if $t \in K_{\mathbf{L}}$,

$$
\varphi(t):=\left\{\begin{array}{l}
t, \quad t \in K_{0}, \\
\infty, \quad t \in K_{\mathbf{L}} \backslash K_{0} .
\end{array}\right.
$$

In order to see that $\varphi$ is continuous, since it is clearly so in the points of $S$, which are isolated, we only need proving it for the points of $\left\{l_{L}: L \in \mathbf{L}\right\} \cup\{\infty\}$. If $t_{0}=l_{L}, L \in \mathbf{L}$, we consider two possibilities

One: $t_{0}=l_{L}$, with $L=L_{\delta}, \delta \in \omega_{1}^{(1)} \backslash \omega_{1}^{(2)}$. Then, $L \in \mathbf{L}_{0}$ and $t_{0}=l_{L} \in K_{0}$. So, $\varphi\left(t_{0}\right)=t_{0}$. Thus, if $V$ is a neighborhood of $t_{0}$ in $K_{\mathbf{L}}$, it contains a set of the form $B \cup\left\{l_{L}\right\}$, where $B$ is a cofinite subset of $L \subseteq \cup \mathbf{L}_{0} \subseteq K_{0}$, and we have

$$
\varphi\left(B \cup\left\{l_{L}\right\}\right)=B \cup\left\{l_{L}\right\} \subseteq V .
$$

Two: $t_{0}=l_{L}$, with $L=L_{\delta}, \delta \in \omega_{1}^{(2)}$. Now, since $t_{0} \notin K_{0}, \varphi\left(t_{0}\right)=\infty$. But $L_{\delta} \subseteq \omega_{1}^{(1)}$ and $\cup \mathbf{L}_{0} \subseteq \omega_{1} \backslash \omega_{1}^{(1)}$ imply that $L_{\delta} \cap K_{0}=\emptyset$, and so $\varphi\left(L \cup\left\{l_{L}\right\}\right)=\{\infty\}$. 
The continuity of $\varphi$ at $t_{0}=\infty$ is clear, since, for any neighborhood $V$ of $\varphi\left(t_{0}\right)=\infty$ in $K_{\mathbf{L}}$, we have

$$
\varphi(V)=\varphi\left(\left(V \cap K_{0}\right) \cup\left(V \backslash K_{0}\right)\right)=\left(V \cap K_{0}\right) \cup\{\infty\} \subseteq V .
$$

To show that $C\left(K_{\mathbf{L}}\right)$ is not extensible, it will be sufficient to see that there exists a copy of $c_{0}\left(\omega_{1}\right)$ which is not complemented. For this, simply consider the closed subspace $Y:=\overline{\operatorname{span}}\left\{1_{\alpha}: \alpha \in \cup \mathbf{L}\right\}$. It can be easily seen that $Y$ is isomorphic to $c_{0}\left(\omega_{1}\right)$ and, if $Y$ were complemented in $C\left(K_{\mathbf{L}}\right)$, then, since $Y=C_{\left\{l_{L}: L \in \mathbf{L}\right\} \cup\{\infty\}}\left(K_{\mathbf{L}}\right)$, we would have

$$
C\left(K_{\mathbf{L}}\right) \simeq Y \times C\left(\left\{l_{L}: L \in \mathbf{L}\right\} \cup\{\infty\}\right) \simeq c_{0}\left(\omega_{1}\right) \times c_{0}\left(\omega_{1}\right) .
$$

A contradiction, since $K_{\mathbf{L}}$ is not an Eberlein compact.

Notice from what we have seen above that $C\left(K_{\mathbf{L}}\right)$, where $\mathbf{L}$ is a non-trivial $\omega_{1}$-ladder system, always contains an uncomplemented copy of $c_{0}\left(\omega_{1}\right)$ given by the subspace $\overline{\operatorname{span}}\left\{1_{\alpha}: \alpha \in \cup \mathbf{L}\right\}=C_{\left\{l_{L}: L \in \mathbf{L}\right\} \cup\{\infty\}}\left(K_{\mathbf{L}}\right)$.

Question 1. Is it so that, for every Mrówka compact $K_{\mathbf{L}}$, where $\mathbf{L}$ is a non-trivial

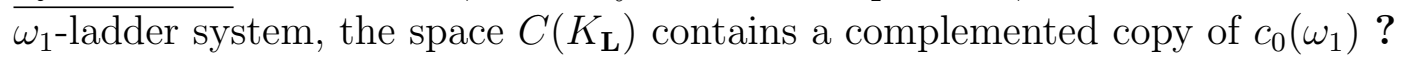

We finish giving a partial answer to the formerly posed question by imposing a condition on an $\omega_{1}$-ladder system $\mathbf{L}$ which, generalizing the situation of Example 3.4, yields a space $C\left(K_{\mathbf{L}}\right)$ containing a complemented copy of $c_{0}\left(\omega_{1}\right)$, and consequently not being extensible. We begin by fixing some particular notation.

If $A$ is set of countable ordinals, $A^{(1)}$ will denote the set of order-accumulation points of $A$. By a counbounded club in $\omega_{1}^{(1)}$ we mean an order-closed unbounded subset $F$ of $\omega_{1}^{(1)}$ such that $\omega_{1}^{(1)} \backslash F$ is not bounded. If $A$ is a subset of $\omega_{1}^{(1)}$, then we put $\mathbf{L}_{A}$ to represent the subfamily of $\mathbf{L}$ given by

$$
\mathbf{L}_{A}:=\left\{L_{\delta}: \delta \in A\right\} .
$$

Clearly, $\cup \mathbf{L}_{A}$ will stand for $\cup_{\delta \in A} L_{\delta}$. By a cofinite refinement of $\mathbf{L}=\left\{L_{\delta}: \delta \in S\right\}$ we mean a collection $\mathbf{R}=\left\{R_{\delta}: \delta \in S\right\}$ such that, for each $\delta \in S, R_{\delta}$ is a cofinite subset of $L_{\delta}$; it is easy to check that the Mrówka compact $K_{\mathbf{R}}$ is homeomorphic to $K_{\mathbf{L}}$.

Lemma 3.5. For each counbounded club $F$ in $\omega_{1}^{(1)}$, there is a cofinite refinement $\mathbf{R}$ of $\mathbf{L}$ such that, for each $\delta \in \omega_{1}^{(1)} \backslash F$,

$$
R_{\delta} \cap\left(\cup \mathbf{R}_{F \backslash F^{(1)}}\right)=\emptyset .
$$

Proof. We construct a cofinite refinement $\mathbf{R}$ of $\mathbf{L}$ such that, if $\mathbf{R}=\left\{R_{\delta}: \delta \in \omega^{(1)}\right\}$, then, given $\delta \in \omega_{1}^{(1)} \backslash F$ and $\gamma \in F \backslash F^{(1)}$, we have $R_{\delta} \cap R_{\gamma}=\emptyset$.

Recall that, for $\delta \in \omega_{1}^{(1)} \backslash F$, there is a unique $\lambda \in F \cup\{0\}$ such that $\left.\delta \in\right] \lambda, \hat{\lambda}[$, where $\hat{\lambda}:=\min \{\beta \in F: \beta>\lambda\}$ (with the convention $\hat{0}:=\min (F)$ ). Similarly, for $\delta \in F \backslash F^{(1)}$, there is a unique $\lambda \in F$ such that $\hat{\lambda}=\delta$. Thus, if $\delta \in \omega_{1}^{(1)}$, we 
define

$$
R_{\delta}:=\left\{\begin{array}{lll}
\left.L_{\delta} \cap\right] \lambda, \delta\left[\backslash L_{\hat{\lambda}},\right. & \text { if } \delta \notin F & (\delta \in] \lambda, \hat{\lambda}[), \\
\left.L_{\delta} \cap\right] \lambda, \delta[, & \text { if } \delta \in F \backslash F^{(1)} & (\delta=\hat{\lambda}), \\
L_{\delta}, & \text { if } \delta \in F^{(1)} &
\end{array}\right.
$$

It is obvious that $\mathbf{R}:=\left\{R_{\delta}: \delta \in \omega_{1}^{(1)}\right\}$ is a cofinite refinement of $\mathbf{L}$. Now, if $\delta \in \omega_{1}^{(1)} \backslash F$ and $\gamma \in F \backslash F^{(1)}$, let $\lambda, \mu \in F$ be such that $\left.\delta \in\right] \lambda, \hat{\lambda}[$ and $\gamma=\hat{\mu}$. Since $\delta \neq \gamma$, we consider two possibilities

One. $\gamma<\delta$. Then, $\mu<\gamma \leq \lambda<\delta$ and so

$$
\left.R_{\gamma} \cap R_{\delta} \subseteq\right] \mu, \gamma[\cap] \lambda, \delta[=\emptyset .
$$

Two. $\delta<\gamma$. Now, $\lambda<\delta<\hat{\lambda} \leq \gamma=\hat{\mu}$ and we must consider two subcases

1) $\hat{\lambda}=\gamma$. Here

$$
R_{\gamma} \cap R_{\delta} \subseteq R_{\gamma} \backslash L_{\hat{\lambda}}=R_{\gamma} \backslash L_{\gamma}=\emptyset .
$$

2) $\hat{\lambda}<\gamma$. In this case $\mu<\delta<\hat{\lambda} \leq \mu<\gamma=\hat{\mu}$, from where we have

$$
\left.R_{\delta} \cap R_{\gamma} \subseteq\right] \lambda, \delta[\cap] \mu, \gamma[=\emptyset .
$$

For each counbounded club $F$ in $\omega_{1}^{(1)}$ and each $\beta \in \omega_{1}$, since $F^{(\beta)}$ is also a counbounded club in $\omega_{1}^{(1)}$, after the former lemma, there exists a cofinite refinement $\mathbf{R}(F, \beta)=\left\{R_{\delta}^{F, \beta}: \delta \in \omega_{1}^{(1)}\right\}$ of $\mathbf{L}$ such that, for each $\delta \in \omega_{1}^{(1)} \backslash F^{(\beta)}$,

$$
R_{\delta}^{F, \beta} \cap\left(\cup \mathbf{R}(F, \beta)_{F^{(\beta)} \backslash F^{(\beta+1)}}\right)=\emptyset .
$$

We may thus make the following definition: A counbounded club $F$ in $\omega_{1}^{(1)}$ is said to be $\mathbf{L}$-stable whenever there is a cofinite refinement $\mathbf{R}$ of $\mathbf{L}$ and an ordinal $\beta \in \omega_{1}$ such that, for each $\gamma \geq \beta$, $\mathbf{R}$ refines $\mathbf{R}(F, \gamma)$. By stab $(\mathbf{L})$ we denote the collection of all $\mathbf{L}$-stable counbounded clubs in $\omega_{1}^{(1)}$.

Question 2. Is it true that, for each $\mathbf{L}, \quad \operatorname{stab}(\mathbf{L}) \neq \emptyset$ ?

Proposition 3.6. If stab $(\mathbf{L}) \neq \emptyset$, then $C\left(K_{\mathbf{L}}\right)$ contains a complemented copy of $c_{0}\left(\omega_{1}\right)$, thus being non-extensible:

Proof. Assuming $F \in \operatorname{stab}(\mathbf{L})$, let $\mathbf{R}$ and $\beta \in \omega_{1}$ be such that, for each $\gamma \geq \beta$, $\mathbf{R}$ refines $\mathbf{R}(F, \gamma)$. If $\mathbf{R}=\left\{R_{\delta}: \delta \in \omega_{1}^{(1)}\right\}$, then, for each $\delta \in \omega_{1}^{(1)} \backslash F^{(\beta)}$, since $\delta \in \omega_{1}^{(1)} \backslash F^{(\gamma)}, \gamma \geq \beta$, we have

$$
R_{\delta} \cap\left(\cup \mathbf{R}_{F^{(\beta)}}\right)=R_{\delta} \cap\left[\bigcup_{\gamma \geq \beta}\left(\cup \mathbf{R}_{F^{(\gamma)} \backslash F^{(\gamma+1)}}\right)\right] \subseteq \bigcup_{\gamma \geq \beta}\left[R_{\delta}^{F, \gamma} \cap\left(\cup \mathbf{R}(F, \gamma)_{F^{(\gamma)} \backslash F^{(\gamma+1)}}\right)\right]=\emptyset .
$$

Putting $H:=F^{(\beta)}$, we have obtained that

$$
\left(\cup \mathbf{R}_{\omega_{1}^{(1)} \backslash H}\right) \cap\left(\cup \mathbf{R}_{H}\right)=\emptyset .
$$

Consequently,

$$
\left\{l_{\delta}: \delta \in H\right\} \cap\left(\overline{\cup \mathbf{R}_{\omega_{1}^{(1)} \backslash H}}\right)=\emptyset .
$$


This means that the set

$$
G:=\left(\cup \mathbf{R}_{\omega_{1}^{(1)} \backslash H}\right) \cup\left\{l_{\delta}: \delta \in \omega_{1}^{(1)} \backslash H\right\}
$$

is almost-clopen in $K_{\mathbf{L}}$, i.e., open and $\bar{G}=G \cup\{\infty\}$. Thus, if $X:=C_{\infty}\left(K_{\mathbf{L}}\right)$, defining $P: X \rightarrow X$ such that $P x:=x \cdot 1_{G}$, we obtain a bounded projection on $X$ which satisfies that $P(X)$ is isomorphic to $C_{\infty}(\bar{G})$. But

$$
\bar{G}=\left(\cup \mathbf{R}_{\omega_{1}^{(1)} \backslash H}\right) \cup\left\{l_{\delta}: \delta \in \omega_{1}^{(1)} \backslash H\right\} \cup\{\infty\}
$$

is a trivial $\omega_{1}$-ladder system compact, therefore $C_{\infty}(\bar{G})$ is isomorphic to $c_{0}\left(\omega_{1}\right)$.

Notice that an affirmative answer to Question 2 would then yield an affirmative answer for Question 1.

Acknowledgement. The author has been partially supported by MICINN Project MTM2011-22417.

\section{REFERENCES}

1. A.V. Arkhangel'skii, Topological Function Spaces, Kluwer, 1992.

2. R.M. Aron, C. Boyd, R.A. Ryan and I. Zalduendo, Zeros of Polynomials on Banach Spaces: The Real Story, Positivity 7 (2003), 285-295.

3. A. Avilés and Y. Moreno, Automorphisms in spaces of continuous functions on Valdivia compacta, Topology Appl. 155 (2008), 2027-2030.

4. T. Banakh, A. Plichko and A. Zagorodnyuk, Zeros of quadratic functionals on non-separable spaces, Colloq. Math. 100 (2004), no. 1, 141-147.

5. R. Deville, G. Godefroy and V. Zizler, Smoothness and renormings in Banach spaces, Pitman Monographs and Surveys in Pure and Applied Math., 64, Longman Sci. and Tech., Harlow, 1993.

6. J. Ferrer, On the Controlled Separable Projection Property for some $C(K)$ spaces, Acta Math. Hung. 124 (2009), no. 1-2, 145-154.

7. J. Ferrer, P. Koszmider and W. Kubiś, Almost disjoint families of countable sets and separable complementation properties, J. Math. Anal. Appl. 401 (2013), 939-949.

8. J. Ferrer and M. Wójtowicz, The Controlled Separable Projection Property for Banach spaces, Cent. Eur. J. Math. 9 (2011), 1252-1266.

9. A. González and V. Montesinos, A note on weakly Lindelöf determined Banach spaces, Czechoslovak Math. J. 59(134) (2009), no. 3, 613-621.

10. T. Jech, Set Theory, The third millennium edition, in: Springer Monographs in Mathematics, Springer-Verlag, Berlin, 2003.

11. J. Kakol, W. Kubiś and M. López-Pellicer, Descriptive Topology in Selected Topics of Functional Analysis, Springer 2011.

12. O. Kalenda and W. Kubiś, Complementation in spaces of continuous function on compact lines, J. Math. Anal. Appl. 386 (2012), 241-257.

13. Y. Moreno and A. Plichko, On automorphic Banach spaces, Israel J. Math. 169 (2009), $29-45$.

14. M. Wójtowicz, Generalizations of the $c_{0}-\ell_{1}-\ell_{\infty}$ theorem of Bessaga and Pelczynski, Bull. Polish Acad. Sci. Math. 50 (2002), no. 4, 373-382.

Department of Mathematical Analysis, University of Valencia, Dr. Moliner 50, Burjassot 46100, Spain.

E-mail address: Jesus.Ferrer@uv.es 